\title{
THE DISTANCE TO THE CENTER OF THE GALAXY
}

\author{
M. J. Reid, M. H. Schneps, J. M. Moran, C. R. Gwinn \\ Harvard-Smithsonian Center for Astrophysics
}

R. Genzel

University of California, Berkeley

D. Downes

Institut de Radio Astronomie Millimetrique

B. Ronnang

Onsala Space Observatory

The distance to a star forming region can be determined by measuring the proper motions within $\mathrm{H}_{2} \mathrm{O}$ maser clusters. If the motions of the maser spots are random, the distance can be determined by applying the technique known as statistical parallax. Alternatively, if organized motions are evident in the proper motions, one can model the source to estimate its the distance. Both methods rely on a comparison of the radial component of the motion (in $\mathrm{km} / \mathrm{s}$ ) and the proper motion on the plane of the sky (in milli-arcseconds/year).

Velocity components toward the observer are determined from Doppler shifts, and angular motions across the plane of the sky are measured by VLBI imaging. Since, in a statistical sense, the transverse velocities should equal the line of sight velocities, the distance to the cluster can be determined by comparison of the angular and line-of-sight velocities. Results for the $\mathrm{H}_{2} \mathrm{O}$ masers associated with Orion A (Genzel et al., 1981, Ap. J., 244, 884) and with W51 (Genzel et al., 1981, Ap. J., 247, 103; Schneps et al., 1981, Ap. J., 249, 124) $\mathrm{H}_{2} \mathrm{O}$ masers have demonstrated the power of this technique.

We have monitored the motions of many $\mathrm{H}_{2} \mathrm{O}$ maser clumps associated with star forming regions in the Galaxy with five VLBI observations from December 1980 through June 1982. In this paper we present results from the $\mathrm{H}_{2} \mathrm{O}$ masers associated with the Sgr B2-North molecular cloud. Relative positional accuracies of about $10 \mu$ as have been achieved for $\mathrm{H}_{2} \mathrm{O}$ masers across a 2 arcsec field, yielding relative transverse velocities with uncertainties of about $3 \mathrm{~km} / \mathrm{s}$ over a 3 month time span.

The proper motion information suggests that the Sgr B2-North masers are expanding. We have modeled the source as a uniformly expanding flow and estimated the parameters including the source distance, the expansion center, and the expansion velocity by fitting the kinematic data in a least-squares sense. 
The expansion center is near the densest concentration of $\mathrm{H}_{2} \mathrm{O}$ features and the expansion velocity is approximately $40 \mathrm{~km} / \mathrm{s}$. This outflow is comparable in linear size but more energetic than the outflow associated with IRc 2 in Orion.

The distance to the Sgr B2-North $\mathrm{H}_{2} \mathrm{O}$ masers is estimated to be $7.1 \mathrm{kpc}$ with a formal uncertainty of $\pm 1.2 \mathrm{kpc}$. We are currently studying sources of systematic error.

The following facts strongly suggest that Sgr B2 is within a few hundred parsecs of the Galactic Center: 1) it is a unique cloud projected only 80 pc from the G.C.; 2) its LSR velocity of $53 \mathrm{~km} / \mathrm{s}$ rules out a chance projection since a velocity near zero would be expected; 3) atomic and molecular absorption studies place Sgr B2 inside the 270 pc expanding shell. Therefore, our results for Sgr B2North suggest a value for $R_{o}$ considerably less than the IAU adopted value of 10 kpc.

Previous studies by our group indicated distances to the Orion masers of $0.48 \pm 0.08 \mathrm{kpc}$ and to the W51 masers of $7 \pm 1.5 \mathrm{kpc}$. Orion is too close to be used to determine $R_{o}$. The W51 result can be used to determine $R_{o}$, provided a kinematic model of the Galaxy is assumed. If $\Theta_{o}=250 \mathrm{~km} / \mathrm{s}$ and $R_{o}=7.1$ $\mathrm{kpc}, \mathrm{W} 51$ 's $v_{L S R}=57 \mathrm{~km} / \mathrm{s}$ implies a distance of $5.5 \mathrm{kpc}$; for $\Theta_{o}=220 \mathrm{~km} / \mathrm{s} \mathrm{a}$ distance close to $5 \mathrm{kpc}$ is favored (although the LSR velocity is slightly above the tangent point value). In short, our measured distance to W51 is about $1-\sigma$ larger than its kinematic distance for $R_{o}=7.1 \mathrm{kpc}$.

The following is a brief list of items which would be affected by reducing $\left.R_{o}: 1\right)$ decrease kinematic distances; 2 ) reduce the mass of the Galaxy and of the Galactic Center; 3) favor recent revisions of the absolute magnitudes of RR Lyrae variables which in turn affects distances in the Local Cluster, the extragalactic scale, and possibly $\left.H_{o} ; 4\right)$ reduce the total luminosity of X-ray bursts, some of which appear super-Eddington for $R_{o}=10 \mathrm{kpc}$ (see Ebisuzaki et al. 1984, P. A. S. Japan 86, 551).

Improvements in the distance to the Galactic Center will come from further analysis of our present data which includes Sgr B2-Middle and W49-North. In particular, W49 is fortuitously located at $l^{I I}=43^{\circ}$ with an LSR velocity near zero $(\approx 9 \mathrm{~km} / \mathrm{s})$; it is far from the tangent point and an estimate of $\boldsymbol{R}_{o}$ from its distance is not sensitive to details of the kinematic model of the Galaxy. W49 is a very strong source with hundreds of maser features and our data should yield a very precise distance. 